PROCEEDINGS OF THE

AMERICAN MATHEMATICAL SOCIETY

Volume 135, Number 4, April 2007, Pages 1065-1071

S 0002-9939(06)08547-9

Article electronically published on September 26, 2006

\title{
ALGEBRAS GENERATED BY THE DISC ALGEBRA AND BOUNDED HARMONIC FUNCTIONS
}

\author{
ALEXANDER J. IZZO
}

(Communicated by Juha M. Heinonen)

\begin{abstract}
Let $D$ denote the open unit disc, and let $A(D)$ denote the disc algebra. The subsets $E$ of $\partial D$ such that the inclusion $A(D)[f, \bar{f}] \supset C(\bar{D})$ holds for every nonconstant $f \in H^{\infty}(D)$ continuous on $E$, or the inclusion $A(D)[f] \supset C(\bar{D})$ holds for every bounded harmonic nonholomorphic function $f$ on $D$ continuous on $E$, are characterized. In the first case the condition is that $E$ has positive measure, and in the second case that $E$ has full measure in $\partial D$.
\end{abstract}

\section{INTRODUCTION}

Let $D$ denote the open unit disc in the plane, let $C(\bar{D})$ denote the algebra of all complex-valued continuous functions on $\bar{D}$, let $A(D)$ denote the disc algebra (the algebra of holomorphic functions on $D$ that extend continuously to $\bar{D}$ ), and let $H^{\infty}(D)$ denote the algebra of bounded holomorphic functions on $D$. A theorem of E. M. Cirka [4, specialized to the disc, asserts that if $f$ is a function in $C(\bar{D})$ and $f$ is harmonic but not holomorphic on $D$, then the uniformly closed subalgebra $A(D)[f]$ of $C(\bar{D})$ generated by $A(D)$ and $f$ is equal to $C(\bar{D})$. An analogous result for $H^{\infty}(D)$ was proved by Sheldon Axler and Allen Shields [3]: If $f$ is a bounded function on $D$ that is harmonic but not holomorphic, then the uniformly closed subalgebra $H^{\infty}(D)[f]$ of $L^{\infty}(D)$ generated by $H^{\infty}(D)$ and $f$ contains $C(\bar{D})$.

Taken together these theorems of Čirka and Axler and Shields lead one to wonder whether the inclusion $A(D)[f] \supset C(\bar{D})$ holds whenever $f$ is a bounded harmonic nonholomorphic function on $D$. It turns out that this is not the case; in fact it is not even true that $A(D)[f, \bar{f}] \supset C(\bar{D})$ whenever $f \in H^{\infty}(D)$ [12, Theorem 7.6]. However, the author gave in [12, Section 7], conditions under which the inclusions $A(D)[f] \supset C(\bar{D})$ and $A(D)[f, \bar{f}] \supset C(\bar{D})$ do hold in terms of the measure of the subset of $\partial D$ where $f$ is continuous. In the present paper we will show that these results are in a certain sense sharp. The results we are concerned with are the following. (Throughout the paper the phrase "Lebesgue measure" will mean onedimensional Lebesgue measure on $\partial D$.)

Theorem A ([12]). If $f \in H^{\infty}(D)$ is nonconstant and is continuous on a subset of $\partial D$ of positive Lebesgue measure, then $A(D)[f, \bar{f}] \supset C(\bar{D})$.

Received by the editors January 12, 2005 and, in revised form, November 1, 2005.

2000 Mathematics Subject Classification. Primary 46J10, 46J15, 30H05.

(C)2006 American Mathematical Society 
Theorem B ([12]). If $f$ is a bounded harmonic function on $D$ that is nonholomorphic and $f$ is continuous a.e. on $\partial D$ (with respect to Lebesgue measure), then $A(D)[f] \supset C(\bar{D})$.

We will show that in both cases the hypothesis on the set on which $f$ is continuous cannot be weakened. We will also show that the conclusion of Theorem A cannot be strengthened to $A(D)[\bar{f}] \supset C(\bar{D})$. In fact we will show that in a certain sense it is no easier to obtain the inclusion $A(D)[f] \supset C(\bar{D})$ when $f$ is conjugate holomorphic than it is when $f$ is merely harmonic but nonholomorphic. This is perhaps somewhat surprising. Explicitly the results we will prove are the following. (We say that a set $E \subset \partial D$ has full measure in $\partial D$ if $\partial D \backslash E$ has Lebesgue measure zero.)

Theorem 1.1. If $E \subset \partial D$ has Lebesgue measure zero, then there is a nonconstant function $f \in H^{\infty}(D)$ that is continuous on $E$ such that $A(D)[f, \bar{f}] \not \supset C(\bar{D})$.

Theorem 1.2. If $E \subset \partial D$ does not have full measure in $\partial D$, then there is a nonconstant bounded real-valued harmonic function $f$ on $D$ that is continuous on E such that $A(D)[f] \not \supset C(\bar{D})$.

Theorem 1.3. If $E \subset \partial D$ does not have full measure in $\partial D$, then there is a nonconstant function $f \in H^{\infty}(D)$ that is continuous on $E$ such that $A(D)[\bar{f}] \not \supset$ $C(\bar{D})$.

Note that by merging Theorems A and B with Theorems 1.1, 1.2, and 1.3, our results can obviously be rephrased as follows.

Theorem 1.4. Given a subset $E$ of $\partial D$, the inclusion $A(D)[f, \bar{f}] \supset C(\bar{D})$ holds for every nonconstant $f \in H^{\infty}(D)$ that is continuous on $E$ if and only if $E$ has positive Lebesgue measure.

Theorem 1.5. Given a subset $E$ of $\partial D$, the inclusion $A(D)[f] \supset C(\bar{D})$ holds for every bounded harmonic nonholomorphic function $f$ on $D$ that is continuous on $E$ if and only if $E$ has full measure in $\partial D$.

Theorem 1.6. Given a subset $E$ of $\partial D$, the inclusion $A(D)[\bar{f}] \supset C(\bar{D})$ holds for every nonconstant function $f \in H^{\infty}(D)$ that is continuous on $E$ if and only if $E$ has full measure in $\partial D$.

Note that Theorem 1.5 is unaffected if the function $f$ is required to be realvalued. For the proof of Theorem 1.1 we will need some information about the zeros of an $H^{\infty}$ function on the circle. A theorem of Fatou [7] (see also [10, p. 80]) characterizes the zero sets of the disc algebra on the circle as the closed subsets of $\partial D$ of Lebesgue measure zero. We will need the following analogue of this theorem for $H^{\infty}$.

Theorem 1.7. Let $E$ be a subset of $\partial D$. If $E$ has Lebesgue measure zero, then there is a nonzero function in $H^{\infty}(D)$ that is continuous on $E$ and is zero at every point of $E$.

Note that the set $E$ is not assumed to be closed. Note also that the converse of this result is a (weak form of a) well-known fact. The author has been unable to find Theorem 1.7 explicitly stated in the literature although it is an immediate consequence of [6, Theorem 1] (or alternatively of results in [5] and in a special case 
of [11, Theorem 2]). However, there is also a simple direct proof of Theorem 1.7. This proof (which is similar to one of the standard proofs of Fatou's theorem) will be given in Section 3 along with the proofs of Theorems 1.1-1.3. A version of the construction used to prove Theorem 1.7 has also been used by W. Hayman 9]. (The author is indebted to the referee for pointing out this reference.)

Another approach to extending the above-mentioned results of Čirka and Axler and Shields is to interpolate between them. In this way the two results can be united into a single concrete theorem. This is done in the author's papers [13] and 14.

The research in the present paper was carried out while the author was a visitor at Brown University. He would like to thank the Department of Mathematics for its hospitality.

\section{Preliminaries}

We begin by making explicit some conventions already used in the introduction. Throughout the paper we tacitly identify $C(\bar{D})$ with the algebra of uniformly continuous functions on $D$ by identifying each member of $C(\bar{D})$ with its restriction to $D$. Given bounded continuous complex-valued functions $f_{1}, \ldots, f_{n}$ on $D$, we denote by $A(D)\left[f_{1}, \ldots, f_{n}\right]$ the uniformly closed algebra on $D$ generated by $A(D)$ and the functions $f_{1}, \ldots, f_{n}$. We say that a complex-valued function $f$ on $D$ is continuous on a subset $E$ of $\partial D$ if $f$ extends to a continuous function on $D \cup E$. For bounded holomorphic or harmonic functions this condition is equivalent to the condition that the function $f^{*}$ defined almost everywhere on $\partial D$ by taking radial limits is continuous on $E$.

The author has given a characterization of the uniformly closed algebras of bounded continuous functions on a bounded open set $\Omega \subset \mathbb{C}$ that contain $C(\bar{\Omega})$ [12, Theorem 3.1]. The results we are going to prove follow from that characterization. However, since we are dealing with very specific algebras we need only a special case of that characterization. The following result, given as Corollary 7.5 in [12, is sufficient for our purposes.

Proposition 2.1. Suppose $f_{1}, \ldots, f_{n}$ are bounded continuous functions on $D$ such that for almost every point $a$ in $D$ we have $\left(\partial f_{j} / \partial \bar{z}\right)(a) \neq 0$ for some $j$. Let $A=A(D)\left[f_{1}, \ldots, f_{n}\right]$. Then $A \supset C(\bar{D})$ if and only if $z_{1}$ is one-to-one over $D$ on the polynomially convex hull of the closure of the set $\left\{\left(z, f_{1}(z), \ldots, f_{n}(z)\right): z \in D\right\}$ in $\mathbb{C}^{n+1}$.

This result points the way to our proofs of the new results in this paper; the question of whether $A(D)\left[f_{1}, \ldots, f_{n}\right] \supset C(\bar{D})$ is converted into a question about the polynomially convex hull of a certain set. Note that since the results we are going to prove all assert that under certain conditions $A(D)\left[f_{1}, \ldots, f_{n}\right] \not \supset C(\bar{D})$, we need only the "only if" direction of Proposition 2.1. In fact all we need is the following special case whose easy direct proof we give. In this way the proofs of Theorems 1.1-1.3 become entirely independent of [12]. (Of course Theorems 1.4-1.6 do rely on [12.)

Lemma 2.2. Suppose $f_{1}, \ldots, f_{n}$ are bounded continuous functions on $D$, and let $X$ denote the polynomially convex hull of the closure of the set $\left\{\left(z, f_{1}(z), \ldots, f_{n}(z)\right)\right.$ : $z \in D\}$ in $\mathbb{C}^{n+1}$. If there exist holomorphic functions $g_{1}, \ldots, g_{n}$ on $D$ such that $\left\{\left(z, g_{1}(z), \ldots, g_{n}(z)\right): z \in D\right\} \subset X$, then $A(D)\left[f_{1}, \ldots, f_{n}\right] \not \supset C(\bar{D})$. 
Proof. To prove this without using Proposition 2.1, first note that since the disc algebra is generated by the identity function $z$, the algebra $A(D)\left[f_{1}, \ldots, f_{n}\right]$ is generated by the functions $z, f_{1}, \ldots, f_{n}$. Consequently, $A(D)\left[f_{1}, \ldots, f_{n}\right]$ is isometrically isomorphic to the uniform closure of the polynomials in the complex coordinate functions on the set $\left\{\left(z, f_{1}(z), \ldots, f_{n}(z)\right): z \in D\right\} \subset \mathbb{C}^{n+1}$, and the maximal ideal space of the latter algebra can be identified with $X$. Thus we may identify the maximal ideal space of $A(D)\left[f_{1}, \ldots, f_{n}\right]$ with $X$, and then the Gelfand transform of $z$ is identified with $z_{1}$.

If $A(D)\left[f_{1}, \ldots, f_{n}\right] \supset C(\bar{D})$, then in particular $\bar{z}$ is in $A(D)\left[f_{1}, \ldots, f_{n}\right]$. By the above paragraph this gives that $\bar{z}_{1}$ is a uniform limit of polynomials in the complex coordinate functions on $X$. But $\bar{z}_{1}$ cannot be such a limit if $X$ contains the analytic $\operatorname{disc}\left\{\left(z, g_{1}(z), \ldots, g_{n}(z)\right): z \in D\right\}$.

\section{THE PROOFS}

Proof of Theorem 1.7. Let $m$ denote Lebesgue measure on $\partial D$ normalized so that $m(\partial D)=1$, and suppose that $m(E)=0$. Then we can choose a sequence of open sets $U_{1}, U_{2}, U_{3}, \ldots$ each containing $E$ such that $U_{1} \supset U_{2} \supset U_{3} \supset \ldots$ and $m\left(U_{n}\right) \leq 1 /\left(n 2^{n-1}\right)$. For convenience assume $U_{1}=\partial D$. Now define a function $w: \partial D \rightarrow[-\infty, \infty)$ by setting

$$
w(x)= \begin{cases}-n & \text { for } x \in U_{n} \backslash U_{n+1}, \\ -\infty & \text { for } x \in \bigcap_{n=1}^{\infty} U_{n} .\end{cases}
$$

It is immediate that $w$ has the following properties:

(i) $w=-\infty$ on $E$;

(ii) $w$ is continuous on $E$ (i.e., for every real number $M$, the set $w^{-1}([-\infty, M))$ contains a neighborhood of $E$ );

(iii) $w \leq-1$ on $\partial D$;

(iv) $w$ is integrable.

Now define $h$ on $D$ by

$$
h(z)=\frac{1}{2 \pi} \int_{-\pi}^{\pi} \frac{e^{i \theta}+z}{e^{i \theta}-z} w\left(e^{i \theta}\right) d \theta .
$$

Then $h$ is holomorphic on $D$ and Re $h \leq-1$ on $D$. Furthermore, straightforward estimation using the Poisson kernel shows that the above properties of $w$ imply that $\operatorname{Re} h(z)$ goes to $-\infty$ as $z$ approaches $E$. Now the function $g=1 / h$ is a nonzero function in $H^{\infty}(D)$ that is continuous on $E$ and zero at every point of $E$.

Proof of Theorem 1.1. By Theorem 1.7, there is a nonzero function $g \in H^{\infty}(D)$ that is continuous on $E$ and zero at every point of $E$. Let $h$ be an inner function that is discontinuous everywhere on $\partial D$. Let $f=g h$. Then $f$ is a nonconstant function in $H^{\infty}(D)$ that is continuous on $E$ and zero at every point of $E$. At every point of $\partial D$ the cluster set of $h$ contains zero [8, Theorem II.6.6], and hence the same is true of the cluster sets of $f$. Hence the set $\{(z, 0,0): z \in D\}$ is contained in the polynomially convex hull of the closure of the set $\{(z, f(z), \bar{f}(z)): z \in D\}$ in $\mathbb{C}^{3}$. Thus Lemma 2.2 shows that $A(D)[f, \bar{f}] \not \supset C(\bar{D})$.

Proof of Theorem 1.2. By the regularity of Lebesgue measure, there is an open subset $U$ of $\partial D$ that contains $E$ and does not have full measure. Furthermore, 
by adjoining to $U$ a dense open subset of $\partial D$ of small measure, we can take $U$ to be dense in $\partial D$. Now let $f$ be the Poisson integral of the characteristic function of $\partial D \backslash U$. Then $f$ is a nonconstant bounded real-valued harmonic function on $D$. Furthermore, $f$ is continuous on $U$. (The proof that the Poisson integral of a continuous function on $\partial D$ is continuous on $\bar{D}$ given in [10, pp. 18 and 32] can easily be adapted to show that the Poisson integral of a function $g$ in $L^{\infty}(\partial D)$ is continuous at each point of $\partial D$ where $g$ is continuous.) In particular $f$ is continuous on $E$. Since $f$ is zero on a dense subset of $\partial D$ (namely $U$ ) we have that the circle $\{(z, 0):|z|=1\}$ lies in the closure of the set $\{(z, f(z)): z \in D\}$. Hence the disc $\{(z, 0): z \in \bar{D}\}$ lies in the polynomially convex hull $X$ of the closure of $\{(z, f(z)): z \in D\}$. Thus Lemma 2.2 shows that $A(D)[f] \not \supset C(\bar{D})$.

Our proof of Theorem 1.3 relies on the existence of a nonconstant function $f \in$ $H^{\infty}(D)$ such that $f$ is continuous on $E$ and for each point $p \in \partial D$ the cluster set $C_{p}$ of $f$ at $p$ is invariant under complex conjugation (i.e., a point is in $C_{p}$ if and only if its complex conjugate is in $C_{p}$ ). (As in Theorem 1.3, we are assuming $E \subset \partial D$ is not of full measure.) In fact, $f$ can be chosen so that $f(D) \subset D$ and at each point $p \in \partial D$ either $f$ is continuous and real-valued at $p$ or else the cluster set of $f$ at $p$ is $\bar{D}$. In the case when $E$ is not dense in $\partial D$, the desired function was constructed by John Wermer and the author. The author showed this construction to Alec Matheson, and subsequently Matheson and Michael Stessin [15] constructed the desired function in the general case, essentially by reducing it to the case treated by Wermer and the author. The author wishes to thank Wermer and Matheson and Stessin for the roles they played in finding the needed function. For completeness we sketch the argument here. (Matheson and Stessin invoke a theorem of Aleksandrov [1, but that will be avoided here.)

Consider first the case when $E$ is not dense in $\partial D$. In this case, by applying an automorphism of the disc, we may assume that $E$ is contained in the left semicircle $(\partial D)_{l}=\{z \in \partial D: \operatorname{Re} z<0\}$. Choose a sequence of points $\left(e^{i \theta_{j}}\right)$ in the right semicircle $(\partial D)_{r}=\{z \in \partial D: \operatorname{Re} z>0\}$ that is dense in $(\partial D)_{r}$. If $\left(c_{j}\right)$ is a sequence of positive numbers with $\Sigma c_{j}<\infty$, then the singular inner function $g$ defined by

$$
g(z)=\exp \left[-\sum c_{j} \frac{e^{i \theta_{j}}+z}{e^{i \theta_{j}}-z}\right]
$$

is continuous on $(\partial D)_{l}$ and discontinuous at every point of $\overline{(\partial D)_{r}}$, and if the $c_{j}$ are chosen sufficiently small, then $g$ sends $(\partial D)_{l}$ into $(\partial D)_{r}$. Let $h$ be a conformal map of $D$ onto the slit disc $D-\{x \in \mathbb{R}: 0 \leq x<1\}$ that sends $(\partial D)_{r}$ onto the slit. Then the function $f=h \circ g$ has the desired properties.

Now consider the general case. By regularity we may assume without loss of generality that $E$ is open. Let $\chi_{E}$ denote the characteristic function of $E$ in $\partial D$ and let $k$ be the Cauchy transform of the measure $\frac{1}{2 \pi i} \chi_{E} d z$ given by

$$
k(\zeta)=\frac{1}{2 \pi} \int_{-\pi}^{\pi} \frac{\chi_{E}\left(e^{i \theta}\right)}{1-\zeta e^{-i \theta}} d \theta, \quad \zeta \in D .
$$

Let $m$ denote Lebesgue measure on $\partial D$ normalized so that $m(\partial D)=1$. Using the relation between the Cauchy kernel and the Poisson kernel, we get that $\operatorname{Re} k$ has radial limit $\frac{1}{2}\left(\chi_{E}+m(E)\right)$ almost everywhere on $\partial D$, that $k$ maps $D$ into the vertical strip $\left\{z: \frac{1}{2} m(E)<\operatorname{Re} z<\frac{1}{2} m(E)+\frac{1}{2}\right\}$, and that $k$ sends $E$ into 
the vertical line $\left\{\operatorname{Re} z=\frac{1}{2} m(E)+\frac{1}{2}\right\}$. Let $\psi$ be the conformal map of the strip $\left\{z: \frac{1}{2} m(E)<\operatorname{Re} z<\frac{1}{2} m(E)+\frac{1}{2}\right\}$ onto $D$ that sends the line $\left\{\operatorname{Re} z=\frac{1}{2} m(E)+\frac{1}{2}\right\}$ onto $(\partial D)_{l}$. Now set $\alpha=\psi \circ k$. Then $\alpha$ is an inner function on $D$ that is continuous on $E$ and sends $E$ into $(\partial D)_{l}$. By the special case considered earlier, we can choose an $H^{\infty}(D)$-function $\beta$ with $\beta(D) \subset D$, with $\beta$ continuous on $(\partial D)_{l}$ and such that at each point $p \in \partial D$ either $\beta$ is continuous and real-valued at $p$ or else the cluster set of $\beta$ at $p$ is $\bar{D}$. Now the function $f=\beta \circ \alpha$ has the desired properties.

We also need the following lemma. This is contained in the Claim on p. 171 of 2], but for completeness we include the short proof.

Lemma 3.1. Let $Y$ be a compact set in $\mathbb{C}^{2}$ lying over $\partial D$ (that is, such that $Y \subset \partial D \times \mathbb{C}$ ). For each point $z \in \partial D$, let $Y_{z}=\{w \in \mathbb{C}:(z, w) \in Y\}$ (the fiber of $Y$ over $z$ ). If $f$ is a function in $H^{\infty}(D)$ such that for each point $z$ in $\partial D$ the cluster set of $f$ at $z$ is contained in $Y_{z}$, then the set $\{(z, f(z)): z \in D\}$ is contained in the polynomially convex hull of $Y$.

Proof. The hypothesis on the cluster sets of $f$ implies that the closure of $\{(z, f(z))$ : $z \in D\}$ is contained in $\{(z, f(z)): z \in D\} \cup Y$. In particular, the latter set is compact; denote it by $K$. Let $P$ be a polynomial on $\mathbb{C}^{2}$. Then $P$ attains a maximum on $K$. If the maximum occurs at a point of $\{(z, f(z)): z \in D\}$, then the function $z \mapsto P(z, f(z))$ on $D$ attains the maximum at some point and hence is constant; this in turn implies that $P$ is constant on $\{(z, f(z)): z \in D\}$. Consequently, the maximum of $P$ on $K$ must occur on $Y$. Thus $\{(z, f(z)): z \in D\}$ is contained in the polynomially convex hull of $Y$.

Proof of Theorem 1.3. By the Matheson-Stessin result discussed above, there is a nonconstant function $f \in H^{\infty}(D)$ that is continuous on $E$ and such that for each point $p \in \partial D$ the cluster set $C_{p}$ of $f$ at $p$ is invariant under complex conjugation. Because of the conjugate invariance of the cluster sets of $f$ the closure of the set $\{(z, \bar{f}(z)): z \in D\}$ is precisely the set

$$
\{(z, \bar{f}(z)): z \in D\} \cup\left(\bigcup_{z \in \partial D}\{z\} \times C_{z}\right) .
$$

By the above lemma, the set $\{(z, f(z)): z \in D\}$ is contained in the polynomial hull of this set. Thus Lemma 2.2 shows that $A(D)[\bar{f}] \not \supset C(\bar{D})$.

\section{REFERENCES}

[1] A. B. Aleksandrov, Measurable partitions of the circle induced by inner functions, Nauchn. Sem. Leningrad. Otdel. Mat. Inst. Steklov (LOMI), 149 (1986), Issled. Linein. Teor. Funktsii XV, 103-106, 188; translation in J. Soviet Math. 42 (1988), no. 2, 1610-1613. MR0849298 (87i:30065)

[2] H. Alexander and J. Wermer, Several Complex Variables and Banach Algebras, 3rd ed., Springer-Verlag, New York, 1998. MR.1482798 (98g:32002)

[3] S. Axler and A. Shields, Algebras generated by analytic and harmonic functions, Indiana Univ. Math. J. 36 (1987), 631-638. MR0905614 (88h:46102)

[4] E. M. Čirka, Approximation by holomorphic functions on smooth manifolds in $\mathbb{C}^{n}$, Mat. Sb. 78 (1969), 101-123; English transl., Math. USSR Sb. 7 (1969), 95-114. MR0239121 (39:480)

[5] J. Détraz, Algèbres de fonctions analytiques dans le disque, Ann. Sci. École Norm. Sup. 3 (1970), 313-352. MR0435421 (55:8381)

[6] A. M. Davie and A. Stray, Interpolation sets for analytic functions, Pacific J. Math. 42 (1972), 33-37. MR0313517(47:2071)

[7] P. Fatou, Series trigonométriques et séries de Taylor, Acta Math., vol. 30, 1906. 
[8] J. B. Garnett, Bounded Analytic Functions, Academic Press, New York, 1981. MR0628971 (83g:30037)

[9] W. K. Hayman, Identity theorems for functions of bounded characteristic, J. London Math. Soc. (2) $\mathbf{5 8}$ (1998), 127-140. MR.1666094 (99m:30073)

[10] K. Hoffman, Banach Spaces of Analytic Functions, Prentice-Hall, Englewood Cliffs, New Jersey, 1962. MR0133008 (24:A2844)

[11] E. A. Heard and J. H. Wells, An interpolation problem for subalgebras of $H^{\infty}$, Pacific J. Math. 28 (1969), 543-553. MR0243359 (39:4681)

[12] A. J. Izzo, Algebras containing bounded holomorphic functions, Indiana Univ. Math. J. 52 (2003), 1305-1342. MR2010729 (2004h:46055)

[13] A. J. Izzo, Algebras generated by holomorphic and harmonic functions on the disc, Bull. London Math. Soc. 37 (2005), 761-770. MR2164839 (2006d:46063)

[14] A. J. Izzo, Some algebras of bounded functions on the disc, Math. Reports Academy Sci. Canada 27 (2005), 72-75. MR2155659 (2006a:30054)

[15] A. L. Matheson and M. I. Stessin, Cauchy transforms of characteristic functions and algebras generated by inner functions, Proc. Amer. Math. Soc. 133 (2005), 3361-3370. MR2161161

Department of Mathematics and Statistics, Bowling Green State University, BowlING Green, Оhio 43403

E-mail address: aizzo@math.bgsu.edu 